\title{
Pulsed photoacoustic Doppler flow measurements in blood-mimicking phantoms
}

\author{
J. Brunker and P. Beard \\ Department of Medical Physics and Bioengineering, University College London, Gower Street, \\ London, WC1E 6BT, United Kingdom \\ http://www.medphys.ucl.ac.uk/research/mle/index.htm
}

\begin{abstract}
The feasibility of making spatially resolved measurements of blood flow using pulsed photoacoustic Doppler techniques has been explored. Doppler time shifts were quantified via cross-correlation of pairs of photoacoustic waveforms generated within a blood-simulating phantom using pairs of laser light pulses. The photoacoustic waves were detected using a focussed or planar PZT ultrasound transducer. For each flow measurement, a series of 100 waveform pairs was collected. Previous data processing methods involved rejection of poorly correlated waveform pairs; the modal velocity value and standard deviation were then extracted from the selected distribution of velocity measurements. However, the data selection criteria used in this approach is to some extent arbitrary. A new data analysis protocol, which involves averaging the 100 cross-correlation functions and thus uses all of the measured data, has been designed in order to prevent exclusion of outliers. This more rigorous approach has proved effective for quantifying the linear motion of micron-scale absorbers imprinted on an acetate sheet moving with velocities in the range 0.14 to $1.25 \mathrm{~ms}^{-1}$. Experimental parameters, such as the time separation between the laser pulses and the transducer frequency response, were evaluated in terms of their effect on the accuracy, resolution and range of measurable velocities. The technique was subsequently applied to fluid phantoms flowing at rates less than $5 \mathrm{mms}^{-1}$ along an optically transparent tube. Preliminary results are described for three different suspensions of phenolic resin microspheres, and also for whole blood. Velocity information was obtained even under non-optimal conditions using a low frequency transducer and a low pulse repetition frequency. The distinguishing advantage of pulsed rather than continuous-wave excitation is that spatially resolved velocity measurements can be made. This offers the prospect of mapping flow within the microcirculation and thus providing insights into the perfusion of tumours and other pathologies characterised by abnormalities in flow status.
\end{abstract}

Keywords: photoacoustic imaging, pulsed, Doppler, flow, cross-correlation

\section{INTRODUCTION}

Photoacoustic imaging relies upon the use of laser generated ultrasound to produce optical absorption based images of soft tissues [1]. Endogenous image contrast is dominated by haemoglobin on account of its strong optical absorption at visible and near infrared wavelengths. As a consequence, photoacoustic imaging is well suited to providing images of vascular anatomy [2,3]. In addition, by varying the excitation laser wavelength and exploiting the known spectral differences between oxy and deoxy-hemoglobin, measurements of absolute blood oxygen saturation can be made [4]. This ability to characterise the structure and oxygenation status of the vasculature makes the technique well suited to the study of tumours and other pathologies characterised by abnormalities in perfusion and oxygen supply. A further potential functional capability is the measurement of blood velocity using Doppler flowmetry techniques. This would be useful in its own right, for example to study flow in tumour vessels where the tortuous nature of the microvasculature can lead to chaotic and variable blood flow which can inhibit therapeutic response. However, if both blood flow and oxygen saturation can be measured simultaneously there is also the enticing prospect of being able estimate oxygen delivery and thus provide a measure of oxygen consumption - an important physiological parameter that is almost impossible to measure non invasively using other methods without employing contrast agents.

Photoacoustic flow measurements can be made in a manner analogous to conventional pulse echo Doppler ultrasound that is to say by recovering the Doppler frequency, phase or time shift encoded on to photoacoustic waves emitted by moving red blood cells. Unlike Doppler ultrasound however, the detected acoustic signal is emitted by the blood cells as opposed to being weakly reflected from them. This offers significant SNR advantages especially when measuring flow in 
microvessels as these exhibit low echogenicity. Furthermore, Doppler ultrasound measurements of the relatively low flow velocity $(<50 \mathrm{~mm} / \mathrm{s})$ in microvessels can be corrupted by the much larger backscattered signal from the surrounding tissue which can move at comparable speeds due to respiratory or cardiac motion. In photoacoustic Doppler flowmetry this is expected to be less problematic due to the strong optical absorption of blood compared to the constituents of the vessel wall and surrounding tissue.

The basic principles of photoacoustic Doppler flowmetry were first outlined in reference [5]. Subsequently, photoacoustic measurements of flow in a tissue mimicking phantom were obtained by recovering Doppler frequency shifts using CW excitation [6-9]. However, in common with CW Doppler ultrasound, this approach cannot readily provide depth-resolved measurements. The use of pulsed excitation overcomes this limitation, and time-resolved spectral analysis has been successful in quantifying Doppler frequency shifts for velocities between 3.5 and $200 \mathrm{mms}^{-1}$ [10,11]. In this paper we expand on the time-shift method described in our previous publication [12]. Flow velocity is determined by measuring the progressive time shift of the detected photoacoustic signals due to the motion of red blood cells illuminated by a series of laser pulses. A time-correlation method is used and, since this can be applied to segments of the detected time-resolved signal corresponding to a specific depth, spatially resolved measurements can be made. Section 2 describes the underlying principles of the technique and section 3 the experimental methods used to evaluate it by means of blood tissue phantoms. Section 4 discusses the signal processing and methods for estimating the velocity. The experimentally determined velocity measurements, resolution and dynamic range are presented and discussed in section 5 .

\section{PRINCIPLES OF PULSED PHOTOACOUSTIC DOPPLER FLOWMETRY}

The photoacoustic (PA) effect can be exploited to make Doppler flow measurements. Consider a cluster of particles, such as red blood cells, within a vessel as shown in Figure 1. Following pulsed wide-field optical illumination, photoacoustic waves are generated and subsequently detected using a directional ultrasound receiver acoustically coupled to the tissue surface. Since an extended region of the tissue is illuminated due to the diffuse nature of light transport in tissue, the beam width of the receiver, rather than the illuminated volume, defines the region over which the signals are collected. The flowmetry is therefore performed in acoustic-resolution mode, which enables greater measuring depths than are possible with optical-resolution Doppler flowmetry [6-9]. Between successive laser pulses, the motion of particles within the transducer focal region will produce a change $d$ in the distance travelled by the signals to the detector, and this produces a difference $t_{s}$ in the signal arrival times. This measured time shift is related to the speed of sound $c$ in the medium and the angle $\theta$ between the direction of particle motion and the axis of the transducer receive beam:

$$
t_{s}=\frac{d}{c}=\frac{l \cos \theta}{c}
$$

$l$ is the distance travelled by the particles between successive laser pulses, which are separated by a time $T$. The velocity of particles $V$ is then given by

$$
V=\frac{l}{T}
$$

which, using Eq. (1), can then be written

$$
V=\frac{c t_{s}}{T \cos \theta}
$$

This is analogous to the Doppler equation derived for pulse-echo ultrasound [13].

The cross-correlation function can be used to determine the time shift $t_{s}$ between two ultrasound waveforms. For continuous functions, $f$ and $g$, the cross-correlation is defined as:

$$
(f \star g)(t)=\int_{-\infty}^{\infty} f^{*}(\tau) g(t+\tau) d \tau
$$

where $f *$ is the complex conjugate of $f$. 


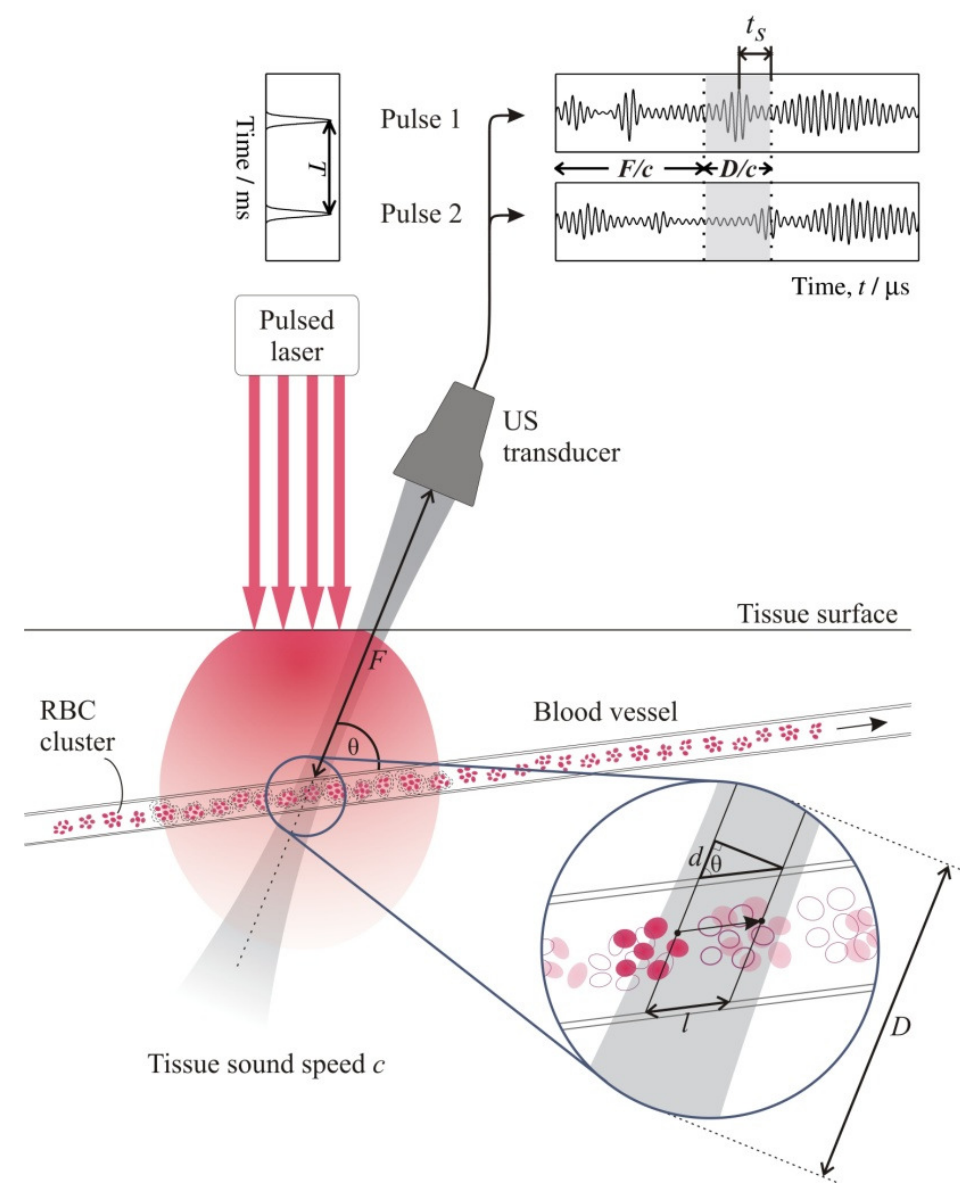

Figure 1. Schematic showing the detection of time-shifted photoacoustic signals. The signals are generated from clusters of moving red blood cells (RBCs) illuminated with an excitation laser pulsing at a frequency 1/T. The insert shows the distribution of RBCs (represented by solid ellipses) when a particular laser pulse is fired, and the new positions (unfilled ellipses) coincident with the firing of the second pulse a time $T$ later. The inset waveforms highlight segments of the two photoacoustic signals that correspond to the depth of the blood vessel and illustrate the time shift $t_{s}$ between the two due to the motion of the RBCs between the two laser pulses.

\section{EXPERIMENTAL METHOD}

Two types of phantom were used to simulate the motion of red blood cells.

The first phantom comprised a wheel mounted on the axle of a DC electric motor. The wheel consists of a Perspex disc overlaid with a sheet of acetate imprinted around the rim with a non-uniform, random pattern of light-absorbing micronscale dots. The distribution of the absorbing dots is shown in Figure 2(a). The dimensions of the dots are on the order of $10 \mu \mathrm{m}$ and they cover approximately $90 \%$ of the total imprinted area. This is comparable to the diameter and volume fraction of red blood cells which are on average $7.5 \mu \mathrm{m}[14]$ and about $50 \%$ respectively.

The second type of phantom was a suspension of phenolic resin microspheres (BRACE GmbH). The solution for suspending the microspheres was made by dissolving an appropriate mass of solid sodium polytungstate (71913, SigmaAldrich) into distilled water so that the mass density of the solution matched that of the microspheres. Three different sizes of microspheres were investigated, with mean particle diameters of $72 \mu \mathrm{m}, 17 \mu \mathrm{m}$ and $6 \mu \mathrm{m}$. Particle aggregation was reduced by adding a 1\% volume of TWEEN® 20 (P1379, Sigma-Aldrich). Fluid flow was generated using a syringe pump (B. Braun Space $\left.{ }^{\circledR}\right)$ with a $60 \mathrm{ml}$ syringe (BD Plastipak ${ }^{\mathrm{TM}}$ ) and a polymer tube (i.d. $800 \mu \mathrm{m}$, Morcap, Paradigm Optics). 


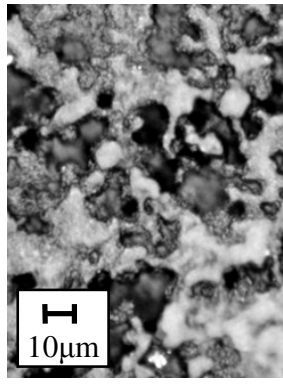

(a) Wheel phantom

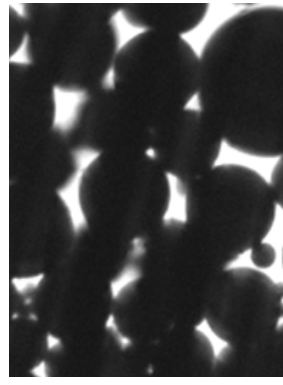

(b) $72 \mu \mathrm{m}$ spheres

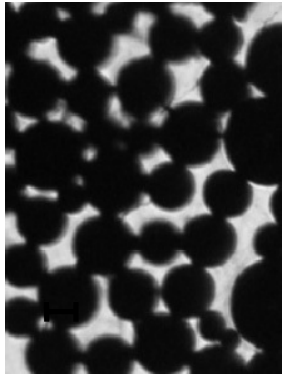

(c) $17 \mu \mathrm{m}$ spheres

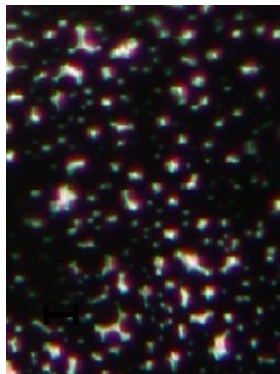

(d) $6 \mu \mathrm{m}$ spheres

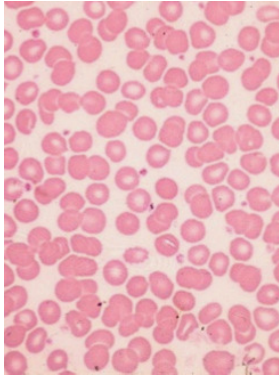

(e) Red blood cells

Figure 2. Comparison of optical microscopy images showing the distribution of absorbers in the wheel phantom (a) and the three fluid phantoms ( $b$ to d), and also a histological section [15] containing red blood cells (e). Each image is shown on the same scale. In the wheel phantom the "particles" comprise micron-scale dots printed onto an acetate sheet, whereas the three fluid phantoms incorporate suspensions of phenolic resin microspheres with mean diameters of 72 $\mu \mathrm{m}, 17 \mu \mathrm{m}$ and $6 \mu \mathrm{m}$.

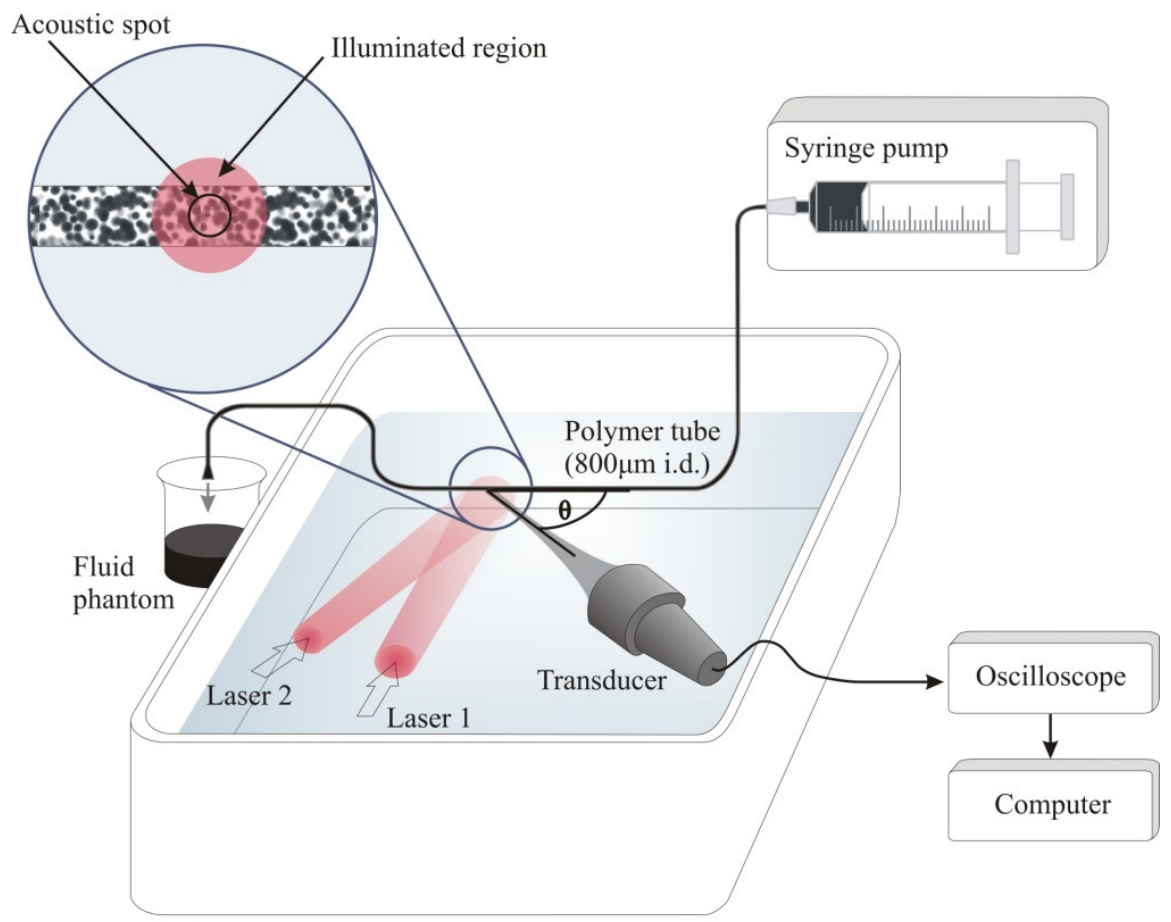

Figure 3. Experimental setup for pulsed photoacoustic Doppler flow measurement where the motion of micron-scale absorbers is used to represent blood flow. Two laser pulses (one emitted by laser 1 and the other by laser 2) separated by a time $T$ are used to generate a pair of acoustic waveforms. Each laser pulse triggers the oscilloscope via a photodiode; the photoacoustic signal received by the ultrasound (US) transducer is acquired on a separate channel, and then downloaded to the computer for processing. The insert shows micron-scale particles with a distribution typical of that found in both the wheel phantom and the fluid phantoms. A large area (at least $2 \mathrm{~cm}$ ) in the locality of the absorbers is illuminated, but photoacoustic signals are only collected from the smaller region (about $1 \mathrm{~mm}$ ) defined by the transducer focal spot.

Figure 3 shows the experimental setup used to demonstrate the technique. Photoacoustic waveform pairs were generated using pairs of laser pulses with pulse separations $T$ in the range $0.1 \mathrm{~ms}$ to $50 \mathrm{~ms}$. In principle, a single laser could be used to produce the pulse pairs. However, since most Q-switched lasers only produce sufficient pulse energies (several mJ) at low, fixed pulse repetition frequencies of a few tens of $\mathrm{Hz}$, the experimental setup employs two Q-switched Nd:YAG 
lasers emitting at $1064 \mathrm{~nm}$. One laser was operated at $20 \mathrm{~Hz}$, and the second laser was triggered off the first after a preselected time delay $T$. Both lasers were carefully aligned in a tank of water so that they illuminated near-identical regions of the phantom. Light absorbing particles within the target area emitted a photoacoustic wave each time they were illuminated and these waves were detected using an ultrasound transducer also immersed in the water. If the position of the particles had shifted between the two laser pulses then a time shift was observed between the two corresponding photoacoustic waveforms. Signals were acquired using an oscilloscope (DSO-Tektronix TDS784D).

\section{SIGNAL ACQUISITION AND PROCESSING}

Series of 100 successive waveform pairs ( 4 x 50 frames of 1000 points in length) were captured in real time using the FastFrame $^{\mathrm{TM}}$ Segmented Memory feature of the oscilloscope. This enabled the waveforms to be concatenated in a single record and downloaded to a PC where the unbiased cross-correlation function was evaluated for each pair of photoacoustic waveforms. The mean and standard deviation of the 100 cross-correlation amplitudes were computed to give a mean cross-correlation function $C(t)$ with error bars equal in length to twice the standard deviation at each point. The peak of $C$ was isolated and the mean of a suitable fit (Gaussian or Weibull) used to determine the measured time shift $t_{s}$ and hence the calculated velocity value $V^{\prime}$ (Eq. (3)). The overall uncertainty $\Delta t_{s}$ in the measured time shift $t_{s}$ was estimated using the standard deviation values (y-axis error bars) associated with $C_{p}$ together with the sensitivity (gradient) of the curve fitting function; the corresponding uncertainty (resolution) $\pm \Delta V^{\prime} / 2$ in the velocity $V^{\prime}$ was calculated using the relation in Eq. (3):

$$
\frac{\Delta V \prime}{2}=\frac{c}{T \cos \theta} \frac{\Delta t_{s}}{2}
$$

Measurements $V^{\prime} \pm \Delta V^{\prime} / 2$ were made for speeds in the range $0.14 \mathrm{~ms}^{-1}$ to $1.25 \mathrm{~ms}^{-1}$ for the wheel phantom, and for 0.14 $\mathrm{mms}^{-1}$ to $7.20 \mathrm{mms}^{-1}$ for the fluid phantoms. Reference [12] describes the method for determining the "known" linear velocity of the rotating wheel at the radius of interest. For the fluid phantom, the flow rate (ml/hr) set on the syringe pump was used to determine the volume of fluid passing through the $800 \mu \mathrm{m}$ diameter tubing, and from this the corresponding velocity was calculated. The "known" values for each phantom were compared with those acquired via cross-correlation of the PA waveform pairs.

\section{RESULTS AND DISCUSSION}

Sub-section 5.1 discusses velocity measurements made using the wheel phantom; preliminary measurements made with the fluid phantom, and also with whole blood, are presented in sub-section 5.2.

\subsection{Wheel phantom}

Results acquired with the wheel phantom for velocities between $0.15 \mathrm{~ms}^{-1}$ and $0.85 \mathrm{~ms}^{-1}$ using a $20 \mathrm{MHz}$ planar transducer (Figure 4(a)) show close agreement with the known values: on average, the measurements are accurate to within approximately $10 \%$ of the known velocity and have a resolution $\Delta V^{\prime} / 2$ of $\pm 0.1 \mathrm{~ms}^{-1}$ (approximately $8 \%$ of the known value). For velocities beyond $0.85 \mathrm{~ms}^{-1}$ the measured values drop to zero due to near-complete loss of correlation between the two waveforms in each pair. This occurs when the absorbers illuminated by the first laser pulse have moved out of the transducer focal region by the time of the second laser pulse. The "drop-off" value defines the maximum measureable velocity, which is determined by the time separation $T$ between the laser pulses and the acoustic spot size of the transducer as described in sub-section 5.1.1. Data acquired using a $3.5 \mathrm{MHz}$ cylindrical focussed transducer (Figure 4(b)) do not show a permanent loss of correlation for velocities within the range of measurements $\left(0.14\right.$ to $\left.1.25 \mathrm{~ms}^{-1}\right)$; although there are three anomalous measurements around $1.0 \mathrm{~ms}^{-1}$ that drop to zero, accuracy is restored for the higher velocity measurements. This capability for measuring higher velocities is due to the fact that this transducer has a larger acoustic spot size than the $20 \mathrm{MHz}$ transducer. However, all the measurements in Figure 4(b) have a poorer resolution (represented by the size of the vertical error bars) than those in Figure 4(a). The limits on measurement resolution are described in sub-section 5.1.2. Before they reach their limits, it is evident from Figure 4 that the measurement accuracy and resolution both become poorer with increasing velocity. This is due to an increasing lack of correlation; in addition, there are likely frequency band limiting effects as discussed in sub-section 5.1.3. 
(a)

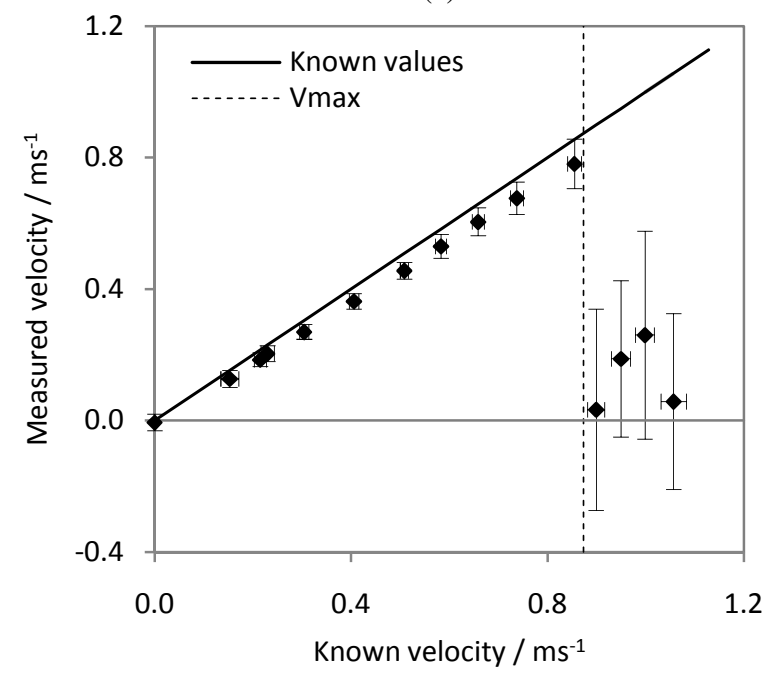

(b)

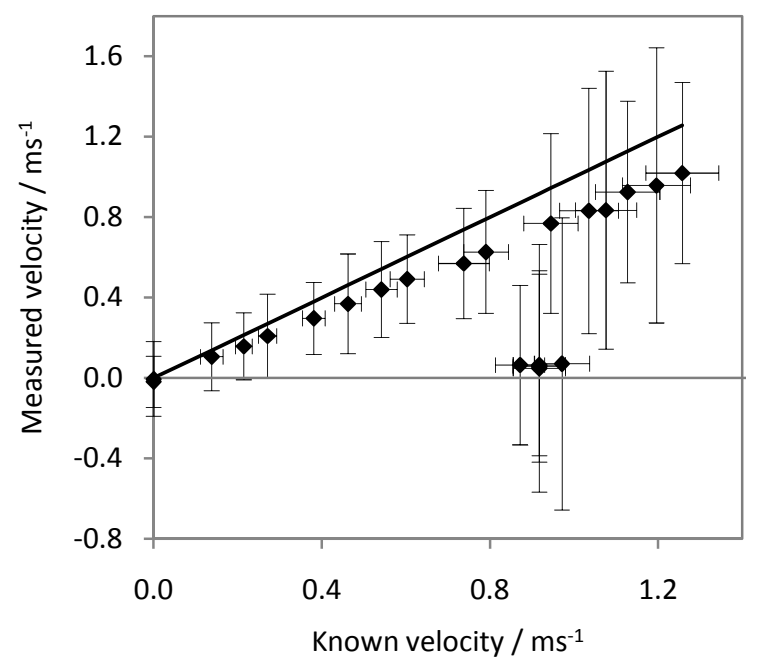

Figure 4. Comparison of velocity values calculated from the time-shifted photoacoustic waveform pairs with the known velocity of the absorbers in the rotating wheel phantom. The time separation between the laser pulses was $1.5 \mathrm{~ms}$. The data in (a) were acquired using a $20 \mathrm{MHz}$ planar transducer. Correlation is lost for velocities beyond that marked by the dashed line. The data in (b) were acquired using a $3.5 \mathrm{MHz}$ cylindrical focussed transducer; the larger focal region prevented complete loss of correlation within the range of measured velocities.

\subsubsection{Upper velocity limits}

Reductions in both accuracy and resolution can be attributed to loss of correlation due to the movement of a particular group of absorbers entirely out of the focal spot by the time the second laser pulse is emitted. The limiting speed $\left|V_{\max }\right|$ (metres per second) beyond which correlation is lost completely can be calculated from the acoustic spot diameter ( $w$ in millimetres) and the pulse separation ( $T$ in milliseconds):

$$
\left|V_{\text {max }}\right|=\frac{w_{p}}{T}=\frac{w}{T \sin \theta}
$$

The geometrical relationship between $w$ and $w_{p}$ is illustrated in Figure 5 . The dashed line in Figure 4 indicates the value for $\left|V_{\max }\right|$ calculated using Eq. (5) for the $20 \mathrm{MHz}$ transducer; this agrees well with the observed "drop-off". The $3.5 \mathrm{MHz}$ transducer has a larger value for $w$ giving rise to a calculated $\left|V_{\max }\right|$ that is beyond the range of velocities measured.

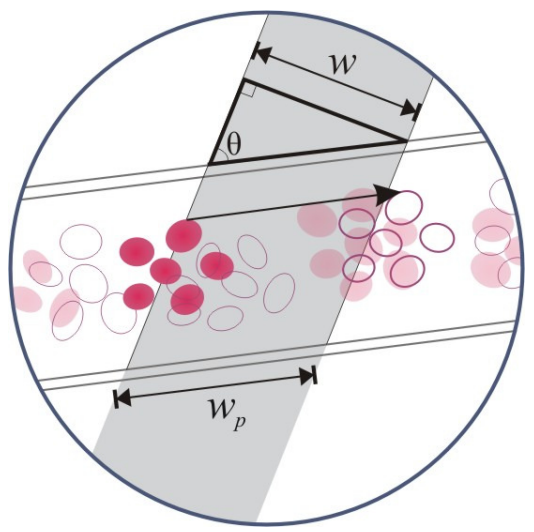

Figure 5. Alignment of the transducer axis at an angle $\theta$ relative to the flow direction. The acoustic spot diameter $w$ results in acquisition of signals from a length $w_{p}$ of the vessel. A clump of RBCs (represented by solid ellipses) may appear within the transducer focal region at the time of the first laser pulse; if the clump moves out of the focal region by the time of the second laser pulse, the speed is greater than $\left|V_{\max }\right|$ and there is no correlation. 


\subsubsection{Resolution limits}

The smallest detectable time shift $\delta t_{s}$ is ultimately limited by the temporal sampling interval of the oscilloscope. If both $c$ and $\theta$ are fixed then, from Eq. (3), it is clear that the smallest detectable velocity $\delta V$ and the time separation $T$ between the laser pulses are inversely proportional:

$$
\delta V \times T=\frac{c \delta t_{s}}{\cos \theta}=\text { constant }
$$

Taking $c=1500 \mathrm{~ms}^{-1}$ and $\theta=50^{\circ}$, then, for $\delta t_{s}=4 \mathrm{ns,}$

$$
\delta V \times T=\frac{1500 \mathrm{~ms}^{-1} \times 4 \times 10^{-9}}{\cos \left(50^{\circ}\right)} \approx 9.33 \times 10^{-6} \mathrm{~m}
$$

This means that for a particular pulse separation $T$ there is a fundamental resolution limit of $\pm \delta V / 2$ :

$$
\frac{\delta V}{2} \approx \frac{4.67 \times 10^{-6} \mathrm{~m}}{T}
$$

A series of measurements were made using seven different pulse separations, and the theoretical resolution $\pm \delta V / 2$ (Eq.(9)) was compared with the measurement resolution $\pm \Delta V / 2$ (Eq.(5)). The results are shown in Figure 6. In the case where the phantom was held stationary, the measurement resolution is approximately twice the fundamental limit $(\delta V / 2)$ at each pulse separation value. For the moving phantom, the measurement resolution was at least three times $\delta V / 2$, and this may be due to variations in the speed of the dc motor. At low pulse separations, the resolution improves in proportion to $1 / T$ and is relatively independent of velocity. As the pulse separation is increased the resolution departs from the inverse relationship: the progressive loss of correlation increases the standard deviation of the 100 crosscorrelation functions used to generate the mean and hence the measurement resolution degrades. For the longest pulse separations where correlation is lost completely, the $1 / T$ dependence appears to be restored.

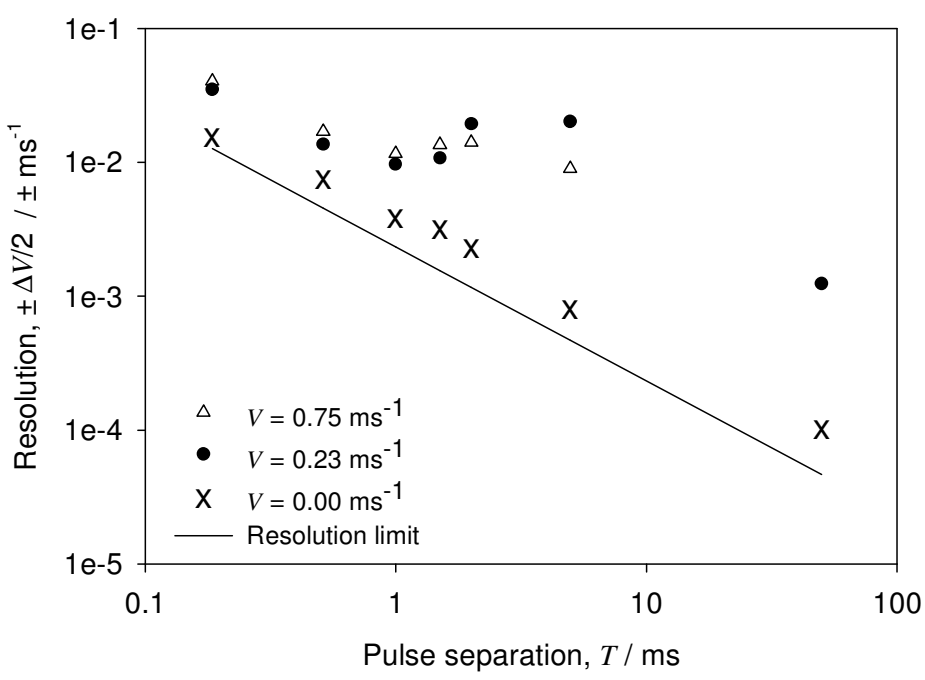

Figure 6. Scalability of velocity resolution with laser pulse separation $T$. The solid line shows the fundamental resolution $\delta V / 2$ determined by the oscilloscope sampling interval $\delta t_{s}$. The symbols show the measurement resolution values $\pm \Delta V^{\prime} / 2$ for three different velocities. Total loss of correlation occurred for the longest pulse separations used with the moving phantom ( $T=50 \mathrm{~ms}$ and $T=5 \mathrm{~ms}$ for $V=0.23 \mathrm{~ms}^{-1}$ and $V=0.75 \mathrm{~ms}^{-1}$ respectively).

\subsubsection{Band limiting effect}

Before they reach the fundamental limits $V_{\max }$ and $\delta V / 2$, the measurement accuracy and resolution are affected by other experimental factors such as the transducer frequency response. A transducer with a low centre frequency reduces the ability to detect the small absorbers responsible for high frequency detail in the PA signals. Moreover, a narrow bandwidth introduces a filtering effect resulting in artificial similarity (correlation) between the PA signals and is likely 
to bias the measurements towards zero time shifts. This zero bias results in measurements that consistently underestimate the known velocity values as shown in Figure 4; thus all the measurements give $A>0$, where the accuracy $A$ is the deviation of the measured velocity $(V$ ') from the known value $(V)$. The accuracy of measurements made at different velocities can be compared using the fractional error:

$$
\frac{A}{|V|}=\frac{|V|-|V \prime|}{|V|}
$$

For speeds below $\left|V_{\max }\right|$, the best results in terms of both accuracy and resolution are therefore likely to be obtained using a broadband transducer centred on a high frequency. Figure 7 compares the accuracy and resolution of the data acquired using $20 \mathrm{MHz}$ and the $3.5 \mathrm{MHz}$ transducers, which are shown in Figure 4. It is evident that the higher frequency, larger bandwidth $20 \mathrm{MHz}$ transducer provides lower fractional errors and higher measurement resolution. When $|V|>\left|V_{\text {max }}\right|$ the fractional error becomes arbitrarily large and the measurement resolution no longer follows a consistent relationship with increasing velocity, pulse separation or transducer frequency.

(a)

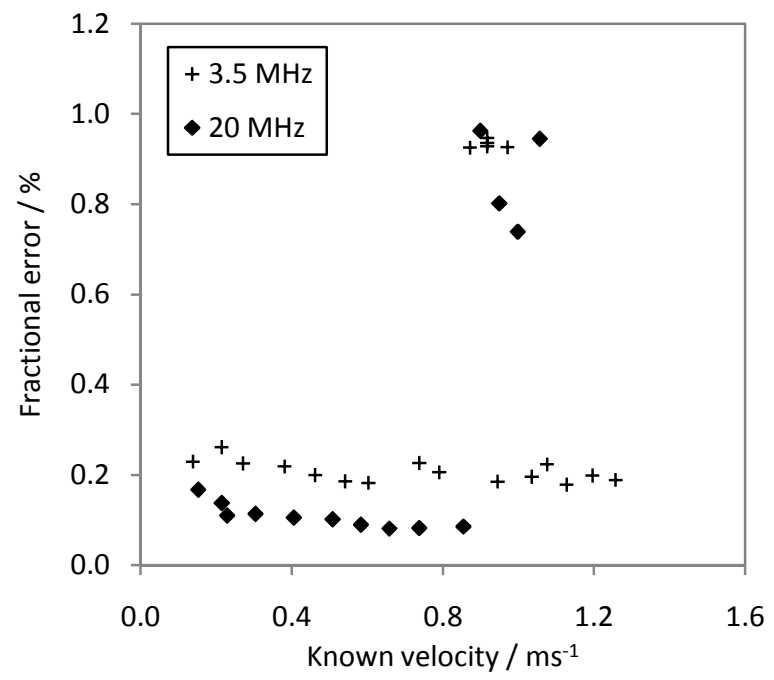

(b)

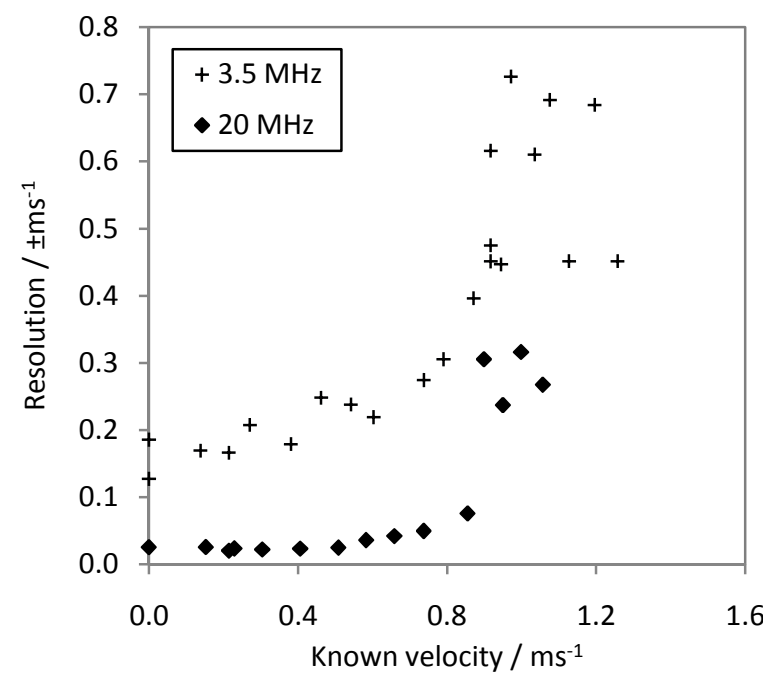

Figure 7. Accuracy (a) and resolution (b) of the data shown in Figure 4. For the data acquired using the $20 \mathrm{MHz}$ transducer, correlation is lost when $\left|V_{\max }\right| \approx 0.85 \mathrm{~ms}^{-1}$ is exceeded. The larger focal region of the $3.5 \mathrm{MHz}$ transducer prevented complete loss of correlation within the range of measured velocities.

\subsection{Fluid phantoms}

Figure 8 shows flow measurements for a suspension of microspheres with average diameter $72 \mu \mathrm{m}$. The nature of flow means that correlation is lost more rapidly in the fluid phantom compared to the rigid wheel phantom. Therefore, a short time separation $T$ between the laser pulses is desirable (Figure 6), as is a high centre frequency, large bandwidth transducer (Figure 7). The preliminary flow measurements in Figure 8 were made using a single laser pulsing at $20 \mathrm{~Hz}$ $(T=50 \mathrm{~ms})$ and a relatively low frequency $(5 \mathrm{MHz})$ transducer. Even under these non-optimal conditions, Figure 8 shows that there is good agreement between the known and measured values for speeds between 0 and $3 \mathrm{mms}^{-1}$, suggesting that a shorter pulse separation and a higher frequency transducer would extend the measurement range and accuracy.

Preliminary measurements were also made using fluid phantoms with mean particle diameters of $17 \mu \mathrm{m}$ and $6 \mu \mathrm{m}$, and also using whole blood, where the mean red cell diameter is taken to be $7.5 \mu \mathrm{m}$ [14]. The measurement range, resolution and accuracy was found to degrade as the particle diameter is reduced, but the similarity of the results for the $6 \mu \mathrm{m}$ particles and for whole blood confirms that this fluid phantom is a realistic representation red cell flow. This suggests that, with appropriate choice of transducer and laser pulse separation, a measurement extent at least comparable to that for the phantom incorporating $72 \mu \mathrm{m}$ particles could be attained for particles of a few microns, and also for whole blood. 


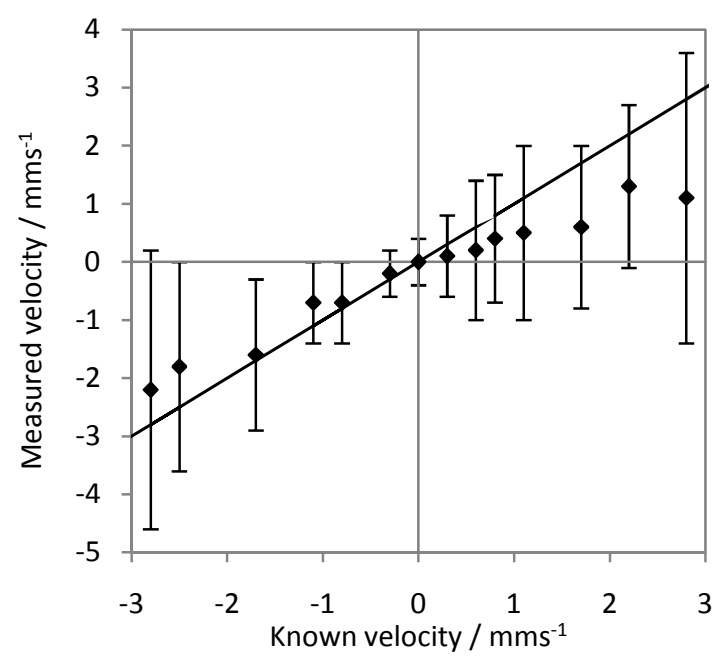

Figure 8. Comparison of velocity values calculated from the time-shifted photoacoustic waveform pairs with the known velocity of the absorbers in the fluid phantom where the mean particle diameter is $72 \mu \mathrm{m}$. These data were acquired using a $5 \mathrm{MHz}$ focussed transducer and the time separation between the laser pulses was $50 \mathrm{~ms}$.

\section{CONCLUSION}

It has been shown that a photoacoustic time shift due to moving particles can be measured via cross-correlation of pairs of photoacoustic waveforms generated using pulsed lasers and detected using an ultrasound transducer. This pulsed photoacoustic Doppler technique has proved effective for making velocity measurements over a range of approximately 0.14 to $1.25 \mathrm{~ms}^{-1}$ using a rotating wheel phantom in which the particle dimensions and densities were comparable to those of red blood cells. Accuracy and resolution appear to be enhanced by using a transducer with a high centre frequency and bandwidth. Moreover, the accuracy and resolution of the velocity measurements are scalable with the time separation between the laser pulses and the acoustic spot size of the transducer. For example, a pulse separation of $50 \mathrm{~ms}$ and acoustic spot size of $0.7 \mathrm{~mm}$ would limit the range to velocities below $18 \mathrm{~mm} / \mathrm{s}$ but give a resolution of $\pm 0.1 \mathrm{~mm} / \mathrm{s}$.; reducing the pulse separation to $5 \mathrm{~ms}$ would allow velocities up to a maximum of about $180 \mathrm{~mm} / \mathrm{s}$ to be measured with a resolution of about $\pm 1 \mathrm{~mm} / \mathrm{s}$.

A laser pulsing frequency of $20 \mathrm{~Hz}$ is insufficient to accurately quantify flow speeds above $3 \mathrm{mms}^{-1}$ in fluid phantoms and whole blood. A $5 \mathrm{MHz}$ transducer also prevents acquisition of optimal flow measurements; the accuracy and resolution of the measurements deteriorate as the diameter of the absorbers is reduced. However, the marked similarity in preliminary results for whole blood and for a suspension of microspheres with a comparable diameter of $6 \mu \mathrm{m}$ warrants a degree of optimism. A short laser pulse separation would extend the range of measurable velocities. A high frequency transducer would enable capture of high frequency PA signals from small diameter structures and it is suggested that a large bandwidth would reduce measurement under-reading due to band-limiting. We anticipate that optimisation of these experimental parameters will allow precise, spatially resolved quantification of low flow speeds such as that of blood in the microcirculation.

\section{REFERENCES}

[1] M. Xu and L.V. Wang, "Photoacoustic imaging in biomedicine," Review of Scientific Instruments, vol. 77, 2006, p. 041101.

[2] E.Z. Zhang, J.G. Laufer, R.B. Pedley, and P.C. Beard, "In vivo high-resolution 3D photoacoustic imaging of superficial vascular anatomy.," Physics in medicine and biology, vol. 54, Feb. 2009, pp. 1035-46.

[3] H.F. Zhang, K. Maslov, G. Stoica, and L.V. Wang, "Functional photoacoustic microscopy for high-resolution and noninvasive in vivo imaging.," Nature biotechnology, vol. 24, Jul. 2006, pp. 848-51. 
[4] J. Laufer, D. Delpy, C. Elwell, and P. Beard, "Quantitative spatially resolved measurement of tissue chromophore concentrations using photoacoustic spectroscopy: application to the measurement of blood oxygenation and haemoglobin concentration.," Physics in medicine and biology, vol. 52, Jan. 2007, pp. 141-68.

[5] P.C. Beard, "Flow velocity measurements," 2001.

[6] J.J. Yao and L.H.V. Wang, "Transverse flow imaging based on photoacoustic Doppler bandwidth broadening," Journal of Biomedical Optics, vol. 15, 2010.

[7] J. Yao, K. Maslov, Y. Shi, L. Taber, and L. Wang, "In vivo photoacoustic imaging of transverse blood flow by using Doppler broadening of bandwidth," Optics Letters, vol. 35, 2010, pp. 1419-1421.

[8] H. Fang, K. Maslov, and L.V. Wang, "Photoacoustic doppler effect from flowing small light-absorbing particles," Physical Review Letters, vol. 99, 2007.

[9] H. Fang, K. Maslov, and L.V. Wang, "Photoacoustic Doppler flow measurement in optically scattering media," Applied Physics Letters, vol. 91, 2007, p. 264103.

[10] A. Sheinfeld, S. Gilead, and A. Eyal, "Simultaneous spatial and spectral mapping of flow using photoacoustic Doppler measurement," Journal of Biomedical Optics, vol. 15, 2010, p. 066010.

[11] A. Sheinfeld, S. Gilead, and A. Eyal, "Photoacoustic Doppler measurement of flow using tone burst excitation," Optics Express, vol. 18, 2010, pp. 4212-4221.

[12] J. Brunker and P. Beard, "Pulsed photoacoustic Doppler flowmetry using a cross correlation method," Proceedings of SPIE, vol. 7564, 2010, pp. 756426-756426-8.

[13] S.G. Foster, P.M. Embree, and W.D. Obrien, "Flow Velocity Profile via Time-Domain Correlation: Error Analysis and Computer Simulation," IEEE Transactions on Ultrasonics Ferroelectrics and Frequency Control, vol. 37, 1990, pp. 164-175.

[14] Bain, B. J., "Morphology of Blood Cells" in Blood Cells: A Practical Guide, Wiley-Blackwell, 52-154 (2002).

[15] "University of Cambridge, Department of Pathology." Available at:

http://www.path.cam.ac.uk/partIB_pract/NHP1/ [Accessed February 8, 2011]. 